\title{
Detection of Actinobacillus pleuropneumoniae ApxIV toxin antibody in serum and oral fluid specimens from pigs inoculated under experimental conditions
}

\author{
Wendy González ${ }^{1}$, Luis G. Giménez-Lirola ${ }^{1}$, Ashley Holmes ${ }^{1}$, Sergio Lizano ${ }^{3}$, Christa Goodell ${ }^{3}$, \\ Korakrit Poonsuk ${ }^{1}$, Panchan Sitthicharoenchai ${ }^{1}$, Yaxuan Sun ${ }^{2}$, Jeffrey Zimmerman ${ }^{1}$ \\ ${ }^{1}$ College of Veterinary Medicine, ${ }^{2}$ College of Liberal Arts and Sciences, \\ Iowa State University, Ames, IA, USA \\ ${ }^{3}$ IDEXX Laboratories, Westbrook, ME, USA \\ jjzimm@iastate.edu
}

Received: January 3, 2017 Accepted: May 26, 2017

\begin{abstract}
Introduction: The prevention and control of Actinobacillus pleuropneumoniae in commercial production settings is based on serological monitoring. Enzyme-linked immunosorbent assays (ELISAs) have been developed to detect specific antibodies against a variety of $A$. pleuropneumoniae antigens, including long-chain lipopolysaccharides (LPS) and the ApxIV toxin, a repeatsin-toxin (RTX) exotoxin unique to A. pleuropneumoniae and produced by all serovars. The objective of this study was to describe ApxIV antibody responses in serum and oral fluid of pigs. Material and Methods: Four groups of pigs (six pigs per group) were inoculated with A. pleuropneumoniae serovars 1, 5, 7, or 12. Weekly serum samples and daily oral fluid samples were collected from individual pigs for 56 days post inoculation (DPI) and tested by LPS and ApxIV ELISAs. The ApxIV ELISA was run in three formats to detect immunlgobulins M, G, and A (IgM, IgG and IgA) while the LPS ELISA detected only IgG. Results: All pigs inoculated with A. pleuropneumoniae serovars 1 and 7 were LPS ELISA serum antibody positive from DPI 14 to 56 . A transient and weak LPS ELISA antibody response was observed in pigs inoculated with serovar 5 and a single antibody positive pig was observed in serovar 12 at $\geq 35$ DPI. Notably, ApxIV serum and oral fluid antibody responses in pig inoculated with serovars 1 and 7 reflected the patterns observed for LPS antibody, albeit with a 14 to 21 day delay. Conclusion: This work suggests that ELISAs based on ApxIV antibody detection in oral fluid samples could be effective in population monitoring for A. pleuropneumoniae.
\end{abstract}

Keywords: Actinobacillus pleuropneumoniae, ApxIV, ELISA, oral fluid, antibody.

\section{Introduction}

Actinobacillus pleuropneumoniae is an important bacterial respiratory pathogen of swine causing acute fibrinohaemorrhagic and necrotising pleuropneumonia (17). In addition, A. pleuropneumoniae is often involved in the porcine respiratory disease complex, a multifactorial infection resulting from the combination of primary and secondary respiratory pathogens $(3,4$, 17, 44). Transmission of $A$. pleuropneumoniae among swine is commonly ascribed to exposure to contaminated respiratory secretions, and/or aerosols (11, 55). More recent publications have described environmental contamination of commercial swine production systems with $A$. pleuropneumoniae, which raises the possibility of indirect routes of transmission (32).
Two A. pleuropneumoniae biotypes have been identified on the basis of the nicotinamide adenine dinucleotide (NAD or V-factor) requirement for growth: biotype I (NAD-dependent) and biotype II (NADindependent) (43). A. pleuropneumoniae is further divided into 15 serovars on the basis of capsular polysaccharide antigen composition $(2,42)$. Recently, a $16^{\text {th }}$ serovar was proposed following the recovery of an APP isolate that did not fit the current classification criteria from swine with pleuropneumonia (6). The degree of virulence among serovars is variable and dependent on several factors, including capsular polysaccharides (CP), lipopolysaccharides (LPS), outer membrane proteins, and the repeats-in-toxin (RTX) exotoxins, e.g. ApxI, II, III, and IV, produced by all serovars albeit in different combinations $(14,22,48)$. 
The Apx toxins are highly immunogenic, i.e. induce antibody production $(14,48)$. Importantly, the ApxIV toxin is unique to $A$. pleuropneumoniae and is produced by all serovars (48). The expression of ApxIV is generally considered to occur only under in vivo conditions (48). Hence, the ApxIV antibody response has been used to differentiate $A$. pleuropneumoniaeinfected animals from those vaccinated with inactivated and/or subunit vaccines (21).

The economic impact of $A$. pleuropneumoniae on the swine industry is a consequence of chronic infections that reduce productivity, i.e. lower feed conversion rates or feed intake; infections that result in acute death, particularly in late finishers; lesions at slaughter; and costs attributable to prevention, control, and/or elimination of $A$. pleuropneumoniae (51). The prevention and control of the A. pleuropneumoniae in commercial production settings is commonly based on the detection of $A$. pleuropneumoniae infections via serological monitoring. Several $A$. pleuropneumoniae serum antibody assays have been described and most are available for use in the diagnostic setting, including the complement fixation (CF) test, a variety of enzyme-linked immunosorbent assays (ELISA), and a fluorescent microsphere immunoassay (15). The CF test is a technically complex assay with poor performance attributes (sensitivity and specificity), for which reasons it is used infrequently (17). In its place, ELISAs have been developed to detect specific antibodies against a variety of $A$. pleuropneumoniae antigens, including capsular polysaccharide (CP), lipopolysaccharide (LPS), and Apx exotoxins (ApxI, II, III, IV) $(18,23,39)$.

Although serum is the historic choice for antibody detection and $A$. pleuropneumoniae surveillance, a variety of antibody-based assays for oral fluid specimens have been reported, e.g. assays for African swine fever virus (ASFV) (38), Erysipelothrix rhusiopathiae (16), influenza A virus (41), porcine circovirus type 2 (45), and porcine reproductive and respiratory syndrome virus (28). The objective of the present study was to describe and compare antibody responses specific for ApxIV in serum and oral fluid from pigs inoculated with $A$. pleuropneumoniae under experimental conditions using a commercial ApxIV antibody ELISA.

\section{Material and Methods}

Experimental design. Four groups of pigs (six pigs per group) were inoculated with $A$. pleuropneumoniae serovars 1, 5, 7, or 12. Serum samples were collected weekly and oral fluid samples were collected daily from individual pigs from the $14^{\text {th }}$ day post inoculation (DPI) through to DPI 56. To confirm infection, serum samples were completely randomised and tested by long-chain lipopolysaccharide (LPS) ELISAs for serovars [1 $(9,11)$, 5a-5b, 7 (4), and 12] at Service de Diagnostic, University of Montreal, Québec, Canada. Serum and oral fluid samples were tested for ApxIV antibody using a commercial assay (ApxIVAb ELISA, IDEXX Laboratories, USA). Statistical analyses were performed to compare the ApxIV responses among and within serovars over time.

Animal management and housing. The 24 14-week-old pigs used in the experiment were housed at the Iowa State University Livestock Infection Disease Isolation Facility, a facility accredited by the Association for Assessment and Accreditation of Laboratory Animal Care. All personnel involved in the experiment had received institutional approval for working with swine and conducting the procedures. Animals were observed a minimum of twice daily throughout the experiment.

Upon arrival, animals were randomly assigned to one of the four $A$. pleuropneumoniae serovar groups by blindly selecting ear tags from a bag. Each consecutive sequence of six ear tag numbers was assigned to one room (serovar). Animals were weighed upon arrival using a portable electronic scale (Pelouze 4040-88 Electronic weighing scale, Rubbermaid Incorporated, USA) for the purpose of assessing general health and group uniformity. Pigs were individually housed in pens equipped with nipple drinkers and fed an antibiotic-free commercial diet (Heartland CO-OP, USA) once daily. Hard plastic balls (Otto Environmental, USA) were provided for enrichment. Pigs were acclimatised for 2 weeks following placement in the facility, during which time they were trained for oral fluid collection. At 58 DPI, pigs were humanely euthanised by penetrating captive bolt (Accles and Shelvoke, UK) followed by exsanguination (31).

Sample collection. To prevent cross-contamination, four people performed daily feeding and oral fluid sampling, i.e. one person for each room (serovar). When it was necessary to move between rooms, e.g. for blood collection, personnel showered and changed coveralls, boots, gloves, masks, and protective eyewear between each room.

Serum samples were collected as mentioned above using a single-use blood collection system (Kendall Company, USA) and then centrifuged at $1,800 \times \mathrm{g}$ for $10 \mathrm{~min}$. Samples were assigned a random number at the time of collection and stored in $2 \mathrm{~mL}$ aliquots at $-80^{\circ} \mathrm{C}$ until tested.

Oral fluid samples were collected daily from individual pigs as described elsewhere $(27,46)$. In brief, a 3-strand, $1.27 \mathrm{~cm}$ diameter, $100 \%$ cotton rope (Grainer Industrial Supply, DSM, USA) was suspended from a bracket fixed to the side of the pen to allow the pigs to chew on the rope. After $30 \mathrm{~min}$, the chewed (wet) end of the rope was inserted into a plastic bag and severed from the dry portion of the rope. To harvest the fluid, the rope was passed through a wringer (WC38K, DynaJetProducts, USA) while in the plastic bag. The fluid that accumulated in the bottom of the bag was then decanted into a $50 \mathrm{~mL}$ centrifuge tube (Corning Inc., USA). Samples were assigned a random number at the time of collection and stored in $4 \mathrm{~mL}$ aliquots at $-80^{\circ} \mathrm{C}$ until tested. 
A total of 203 serum samples were collected from DPI 0 to 56, after which they were completely randomised and tested by 4 LPS ELISAs and the ApxIV ELISA. A total of 1,311 oral fluid samples were collected from DPI -8 to 56 , with a mean sample volume of $8.22 \pm 5.6 \mathrm{~mL}$ (standard deviation (SD)) per pig. A subset of oral fluid samples $(n=1,264)$ were selected, completely randomised, and tested by ApxIV ELISA.

Inoculum preparation. Four strains of A. pleuropneumoniae were used in this experiment: serovar 1 (ATCC 27088), serovar 5a (ATCC 33377), serovar 7 (WF83), and serovar 12 (9499/84). For propagation, the bacteria were cultured on chocolate agar plates (Remel, Inc., USA), incubated at $35^{\circ} \mathrm{C}$ with $5 \%-10 \% \mathrm{CO}_{2}$ for $48 \mathrm{~h}$. To check for purity, bacterial isolates were streaked on 5\% sheep blood agar plates (Remel, Inc.). Plates were then cross-streaked with Staphylococcus epidermidis and incubated at $35^{\circ} \mathrm{C}$ for $48 \mathrm{~h}$.

Confirmation of the $A$. pleuropneumoniae serovars used in this study was performed with slide agglutination tests using rabbit hyperimmune sera against A. pleuropneumoniae reference serovars following standard protocols in place at the Iowa State University Veterinary Diagnostic Laboratory. In brief, bacteria were harvested from chocolate agar plates and mixed with normal saline $(\sim 2.5 \mathrm{~mL})$ to a McFarland turbidity of 0.5 (37). Thereafter, each bacterial suspension was dispensed onto plastic 3-well blood typing plates (Fisherbrand ${ }^{\mathrm{TM}}$, USA) at a rate of three drops per well to which one drop of each serovar-specific serotyping reagent was added (30). After mixing on a mechanical rotator (TekTator V, American Hospital Supply Corp., USA) for $2 \mathrm{~min}$ at $140 \mathrm{rpm}$, the serovar was identified by agglutination of the bacterial suspension with a specific serotyping reagent. Agglutination in more than one well was interpreted as an untypeable response (52).

To prepare the inoculum, the bacteria were harvested from the chocolate agar plate and suspended in normal saline $(\sim 2.5 \mathrm{~mL})$ to a McFarland turbidity of 0.5 . To estimate the bacterial concentration, six ten-fold serial dilutions $\left(10^{-1}\right.$ to $\left.10^{-6}\right)$ were made of the McFarland suspension into sterile saline. A $10 \mu \mathrm{L}$ aliquot of each dilution was pipetted onto a chocolate agar plate and spread on the plate. After $48 \mathrm{~h}$ of incubation, the numbers of colonies were counted and used to estimate the colony forming units (CFU) per millilitre in the McFarland suspension $(35,52)$.

Inoculation and post-inoculation observations. Prior to inoculation, all animals tested negative for A. pleuropneumoniae serum antibody by two commercial ELISAs (ApxIVAb ELISA, IDEXX Laboratories, USA, Swinecheck Mix-APP, Biovet, Inc., Canada) at the Iowa State University Veterinary Diagnostic Laboratory (Ames, USA). To reduce animal stress and facilitate handling, pigs were sedated immediately prior to inoculation using a solution formulated by reconstituting $250 \mathrm{mg}$ of tiletamine and $250 \mathrm{mg}$ of zolazepam (Telazol®, Fort Dodge Animal Health, USA) with $2.5 \mathrm{~mL}$ of xylazine (Lloyd Laboratories, USA) $(100 \mathrm{mg} / \mathrm{mL})$ and $2.5 \mathrm{~mL}$ of ketamine (Fort Dodge Animal Health) $(100 \mathrm{mg} / \mathrm{mL})$. An intramuscular dose of $0.05 \mathrm{~mL} / \mathrm{kg}$ of body weight provided approximately $20 \mathrm{~min}$ of sedation. Each room of animals $(\mathrm{n}=6)$ was inoculated with one A. pleuropneumoniae serovar $(1,5,7$ or 12). Animals were inoculated by instilling $2 \mathrm{~mL}$ of the inoculum intranasally (IN) and then swabbing the tonsils of the soft palate with a 16" large-tip cotton swab (Birchwood Laboratories, Inc., USA) saturated with $3 \mathrm{~mL}$ of the inoculum. Visualisation of the tonsils and the process of tonsil swabbing were facilitated with the use of an oral speculum. For the first $24 \mathrm{~h}$ post inoculation, the animals were observed for clinical signs and rectal temperatures were taken every $3 \mathrm{~h}$ by the same individual. Thereafter, clinical observations were made each morning at the time of oral fluid collection. Any animal exhibiting respiratory distress, emesis, reluctance to stand, and/or a rectal temperature $\geq 41^{\circ} \mathrm{C}$ at two consecutive observations was intramuscularly treated with ampicillin (Polyflex ${ }^{\circledR}$, Boehringer Ingelheim, Vetmedica, USA) at a dose of $5 \mathrm{mg} / \mathrm{kg}$.

Serum antibody testing. To confirm infection, serum samples were tested at the Service de Diagnostic University of Montreal (Québec, Canada) with four long-chain lipopolysaccharide (LPS) ELISAs designed to detect capsular serovars [1 (as well as 9 and 11), 5a-5b, 7 (as well as 4), and 12].

To detect ApxIV immunoglobulin G (IgG) antibody, serum samples were tested at Iowa State University using a commercial indirect ELISA (ApxIVAb ELISA, IDEXX Laboratories, USA). The test was performed according to the manufacturer's instructions and a positive response was defined as a sample-to-positive $(\mathrm{S} / \mathrm{P})$ ratio $\geq 0.5$.

$\mathrm{S} / \mathrm{P}$ ratio $=\frac{(\text { sample OD }- \text { negative control mean OD })}{(\text { positive control mean OD }- \text { negative control mean OD })}$

The manufacturer's protocol was also followed for the detection anti-ApxIV IgM and IgA, but the kit IgG secondary antibody conjugate was replaced with goat anti-pig IgM or IgA horseradish peroxidase-labelled conjugates diluted 1:2,000 and 1:15,000, respectively.

Oral fluid antibody testing. Prior to antibody testing, oral fluid samples were processed to remove suspended particulates by adding $1 \mathrm{ml}$ of sample to a tube containing $100 \mu \mathrm{L}$ of a lyophilised coagulant formulation (ISURF \#04489). The tubes were shaken for $1 \mathrm{~min}$ and centrifuged $\left(4^{\circ} \mathrm{C}\right)$ at $1,200 \times g$ for $3 \mathrm{~min}$. The supernatant was tested for ApxIV antibody with a commercial serum antibody ApxIV ELISA (ApxIVAb ELISA, IDEXX Laboratories, USA) using protocols modified to detect ApxIV IgM, IgA, and IgG in oral fluids. Specifically, ELISA kit serum plate controls were diluted to an OD of 0.1 (negative control) and 1.3 to 1.5 (positive control) and the ELISA kit IgG secondary antibody conjugate was replaced with goat anti-pig IgM, IgG, or IgA horseradish peroxidase-labelled conjugate (Bethyl, USA) diluted to $1: 2,000,1: 3,000$, or 1:15,000, respectively. To perform the test, samples were diluted 1:2 with sample diluent (ApxIVAb ELISA, IDEXX 
Laboratories, USA), $100 \mu \mathrm{L}$ of each sample was added to each well, and the plates were incubated at $37^{\circ} \mathrm{C}$ for $120 \mathrm{~min}$. Plates were then washed five times, $100 \mu \mathrm{l}$ of anti-pig $\operatorname{IgG}, \operatorname{IgM}$, or IgA was added to each well, and the plates were incubated at $37^{\circ} \mathrm{C}$ for $60 \mathrm{~min}$. Thereafter, 3,3',5,5'-tetramethylbenzidine substrate $(100 \mu \mathrm{L})$ was added to each well and the plates were incubated at room temperature for $15 \mathrm{~min}$. Stop solution $(100 \mu \mathrm{L})$ was added to each well, after which the reaction was read at $450 \mathrm{~nm}$ on an ELISA plate reader. The S/P ratios were calculated for each sample using the formula provided by the kit's manufacturer.

Data analysis. Statistical analyses were performed using a commercial package (SAS version 9.4, Institute Inc., USA). Repeated measure analysis of variance with a compound symmetry covariance structure was used to compare oral fluid results $\mathrm{S} / \mathrm{P}$ IgG, S/P IgA, and S/P IgM among the four serovar groups over time. A log transformation was made to the response variables and the multivariate normality assumption was checked by the residual plots. Least squares means were reported for serovar by DPI. If a significant difference among serovars were detected, a post hoc test with Bonferroni adjustment was conducted to compare serovars by DPI. Additionally within each serovar, a response for each DPI was also compared to DPI 0.

\section{Results}

Clinical observations. As shown in Table 1, the frequency with which clinical signs were observed varied among serovar groups. No clinical signs were observed in pigs inoculated with serovar 12 at any time. Some or all of serovar 5 pigs were reluctant to stand through DPI 0 to 4 , but did not exhibit other clinical signs. Pigs inoculated with serovars 1 or 7 exhibited clinical signs in the first $24 \mathrm{~h}$ post inoculation and through to DPI 11, including panting, reluctance to move, vomiting, anorexia, and rectal temperature $\geq 41^{\circ} \mathrm{C}$. All animals from these two groups were treated with ampicillin from DPI 4 through to DPI 11. One pig inoculated with serovar 1 was found dead on the morning of DPI 3. Postmortem examination revealed lesions characteristic of $A$. pleuropneumoniae infection, i.e. tonsils covered with purulent exudate, trachea and bronchi filled with a foamy exudate and extensive pleural adhesions. One pig from the serovar 7 group was humanely euthanised on DPI 20 due to lameness unrelated to $A$. pleuropneumoniae infection.

LPS ELISA serum antibody responses. Serum samples were tested by the four LPS ELISAs, with positive results only observed against the serovar to which animals were exposed. The LPS ELISA serum antibody response varied significantly among serovars (Fig. 1, Table 2). Based on the manufacturer's recommended cutoff of $\mathrm{OD} \geq 0.40$, all pigs exposed to serovars $1(n=5)$ and $7(n=6)$ were positive from DPI 14 through DPI 56. In serovar 5, four of six pigs were positive at DPIs $14,21,28$, then two positive pigs at DPI
35 , and one positive pig at DPI 42 and later. In serovar 12 , pigs were negative by the serovar 12 LPS ELISA, except for one pig that tested positive on DPIs 35,42 , and 49.

Within serovars, comparisons showed that all serovar 1 OD responses at DPI $\geq 7$ were higher than DPI 0 OD responses $(p<0.0001)$. For serovars 5 and 7 , ODs at DPI 14 and later were significantly higher than DPI $0(\mathrm{p}<0.0001)$. For serovar 12 , pairwise comparisons found no difference in mean OD responses when compared to DPI 0 except for DPI $49(\mathrm{p}=0.038)$.

Between serovars, the magnitude of LPS OD responses differed significantly over time $(\mathrm{p}<0.0001)$. Comparisons by DPI detected no difference between serovar 1 and 7 OD responses. At DPIs 14 through 56, serovar 1 and 7 responses were significantly higher than serovars 5 and $12(\mathrm{p}<0.0001)$. At DPIs 14 through 42, serovar 5 OD responses were higher that serovar 12 $(\mathrm{p}<0.0001)$.

ApxIV ELISA serum antibody responses. The ApxIV serum antibody (IgG) ELISA $\mathrm{S} / \mathrm{P}$ responses varied among serovars (Table 3). Based on the manufacturer's recommended cutoff $(\mathrm{S} / \mathrm{P} \geq 0.50)$, three of five pigs in serovar 1 and five of five pigs in serovar 7 became positive over the course of the study. No pigs in serovars 5 or 12 met the manufacturer's criterion for positivity.

Within serovars, all serovars 1 and 7 IgG ELISA $\mathrm{S} / \mathrm{P}$ responses at DPI 21 and later were significantly higher than their DPI $0 \mathrm{~S} / \mathrm{P}$ responses $(\mathrm{p}<0.0001)$. In serovars 5 and 12, pairwise comparisons found no difference in $\mathrm{S} / \mathrm{P}$ responses over time when compared to DPI 0.

A comparison among serovars showed that the serovar $1 \mathrm{IgG} \mathrm{S} / \mathrm{P}$ response was higher than serovars 5 and 12 at DPI 28 and later $(\mathrm{p}<0.0033)$. The serovar $7 \mathrm{IgG} \mathrm{S} / \mathrm{P}$ response was higher than the serovar 1 response at DPI $21(\mathrm{p}=0.0090)$ and $56(\mathrm{p}=0.0301)$ and higher than serovars 5 and 12 at DPI 14 and later $(\mathrm{p}<0.0040)$. No difference was detected in the serovar 5 and 12 responses over time.

Within serovars, the IgM S/P responses were higher than DPI 0 responses at DPI $14(\mathrm{p}<0.0001)$ for serovars 1 and 7 (Table 3 ). In serovars 5 and 12 , no difference was found in IgM responses at any time point when compared to DPI 0. The serovar $7 \mathrm{IgM} \mathrm{S} / \mathrm{P}$ response was higher than serovars 1,5 , and 12 at DPIs 14 and 21 $(\mathrm{p}<0.0020)$. Serovar $1 \mathrm{IgM} \mathrm{S} / \mathrm{P}$ responses differed from serovar 12 at DPI $14(\mathrm{p}=0.0163)$ and DPI 42 $(\mathrm{p}=0.0264)$.

Within serovars, comparisons detected $\operatorname{IgA} \mathrm{S} / \mathrm{P}$ responses higher than DPI 0 at DPI $56(\mathrm{p}=0.0373)$ in pigs exposed to serovar 1. Likewise, detectable differences were found on $\operatorname{IgA}$ responses in pigs exposed to serovars 5 at DPI $35(\mathrm{p}=0.0485)$ and DPI 42 $(\mathrm{p}=0.0015)$. No detectable differences were found on serovars 7 and 12 when compared with DPI 0 . Comparisons among serovars found no detectable difference in the magnitude of $\operatorname{IgA} \mathrm{S} / \mathrm{P}$ responses over time. 
Table 1. Clinical observations following inoculation with Actinobacillus pleuropneumoniae

\begin{tabular}{|c|c|c|c|c|c|c|c|c|c|}
\hline & & \multicolumn{8}{|c|}{ Number of pigs by day post inoculation $^{*}$} \\
\hline & Observation & $0^{\dagger}$ & 1 & 2 & 3 & 4 & 5 & 6 & 7 \\
\hline \multirow{6}{*}{ 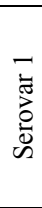 } & Reluctant to stand & 6 & 6 & 6 & 5 & 4 & 4 & 1 & 1 \\
\hline & Respiratory distress & 6 & 6 & 6 & 5 & 5 & 5 & 5 & 5 \\
\hline & Emesis & 5 & 5 & 5 & 5 & 5 & 5 & 5 & 5 \\
\hline & Rectal temperature $\geq 41 \mathrm{C}$ & 1 & •\# & • & • & • & • & - & • \\
\hline & Anorexia & 6 & • & 6 & 5 & 5 & 2 & 2 & 0 \\
\hline & Oral fluid collection $(\geq 1 \mathrm{~mL})$ & 4 & 0 & 0 & 1 & 2 & 3 & 3 & 3 \\
\hline \multirow{6}{*}{ 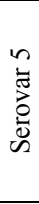 } & Reluctant to stand & 6 & 6 & 6 & 2 & 2 & $\bullet$ & $\bullet$ & • \\
\hline & Respiratory distress & - & • & - & • & - & • & • & - \\
\hline & Emesis & • & • & • & • & • & • & • & - \\
\hline & Rectal temperature $\geq 41 \mathrm{C}$ & • & $\bullet$ & • & $\bullet$ & • & • & $\bullet$ & - \\
\hline & Anorexia & • & • & • & • & • & • & • & • \\
\hline & Oral fluid collection $(\geq 1 \mathrm{~mL})$ & 6 & 3 & 3 & 5 & 5 & 4 & 5 & 4 \\
\hline \multirow{6}{*}{ 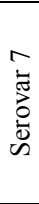 } & Reluctant to stand & 6 & 6 & 6 & 6 & 6 & 6 & 6 & 6 \\
\hline & Respiratory distress & 6 & 6 & 6 & 6 & 6 & 6 & 6 & 6 \\
\hline & Emesis & 6 & 6 & 6 & 2 & 2 & • & • & • \\
\hline & Rectal temperature $\geq 41 \mathrm{C}$ & 1 & • & • & • & • & • & • & • \\
\hline & Anorexia & 6 & 6 & 6 & 6 & 6 & 6 & 6 & 6 \\
\hline & Oral fluid collection $(\geq 1 \mathrm{~mL})$ & 5 & 0 & 0 & 0 & 0 & 3 & 5 & 0 \\
\hline \multirow{6}{*}{ 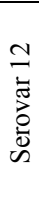 } & Reluctant to stand & $\bullet$ & • & $\bullet$ & $\bullet$ & $\bullet$ & $\bullet$ & $\bullet$ & $\bullet$ \\
\hline & Respiratory distress & $\bullet$ & $\bullet$ & $\bullet$ & $\bullet$ & $\bullet$ & $\bullet$ & $\bullet$ & • \\
\hline & Emesis & • & • & • & • & • & • & • & - \\
\hline & Rectal temperature $\geq 41 \mathrm{C}$ & • & • & • & • & • & • & • & • \\
\hline & Anorexia & • & • & • & • & • & • & • & • \\
\hline & Oral fluid collection $(\geq 1 \mathrm{~mL})$ & 6 & 5 & 6 & 6 & 5 & 6 & 6 & 5 \\
\hline
\end{tabular}

*Denominator is six pigs in all groups at all DPIs, except serovar 1 (one pig died on DPI 2); ${ }^{\dagger}$ First $24 \mathrm{~h}$ following inoculation; \#Symbol $(\bullet)$ indicates not observed in any pigs in the group

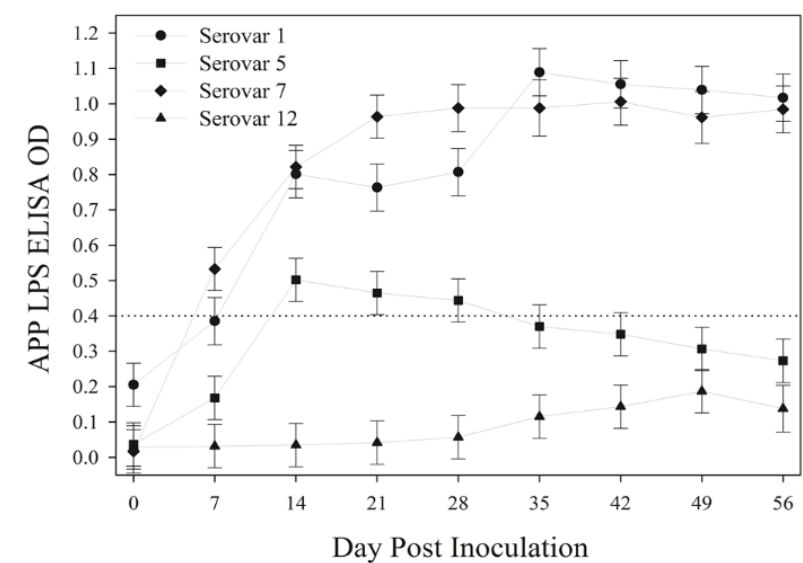

Fig. 1. Actinobacillus pleuropneumoniae quantitative serum antibody responses (least square means) by serovar over time based on serogroupcapsular long-chain lipopolysaccharide (LPS) ELISAs. APP LPS ELISA interpretation is based on optical density (OD): negative $\mathrm{OD}<0.30$, suspect $\mathrm{OD} 0.30-0.39$, positive OD $\geq 0.40$

Table 2. Actinobacillus pleuropneumoniae qualitative serum antibody responses by serovar over time based on serogroupcapsular long-chain lipopolysaccharide (LPS) enzyme-linked immunosorbent assays (ELISAs) ${ }^{*}$

\begin{tabular}{|c|c|c|c|c|c|c|c|c|c|c|c|}
\hline \multirow[b]{2}{*}{ Inoculum } & \multirow[b]{2}{*}{ LPS ELISA } & \multirow[b]{2}{*}{ Pig status } & \multicolumn{9}{|c|}{ Number of pigs by day post inoculation } \\
\hline & & & 0 & 7 & 14 & 21 & 28 & 35 & 42 & 49 & 56 \\
\hline Serovar 1 & $1-9-10-11$ & positive & 1 & 3 & 5 & 4 & 4 & 5 & 5 & 5 & 5 \\
\hline \multirow[t]{3}{*}{ ATCC 27088} & & suspect & 0 & 1 & 0 & 0 & 0 & 0 & 0 & 0 & 0 \\
\hline & & negative & 5 & 1 & 0 & 1 & 1 & 0 & 0 & 0 & 0 \\
\hline & & no. pigs & 6 & 5 & 5 & 5 & 5 & 5 & 5 & 5 & 5 \\
\hline Serovar 5a & $5 a-5 b$ & positive & 0 & 0 & 4 & 4 & 4 & 2 & 1 & 1 & 1 \\
\hline \multirow[t]{3}{*}{ ATCC 33377} & & suspect & 0 & 0 & 1 & 1 & 1 & 3 & 4 & 2 & 1 \\
\hline & & negative & 6 & 6 & 1 & 1 & 1 & 1 & 1 & 3 & 4 \\
\hline & & no. pigs & 6 & 6 & 6 & 6 & 6 & 6 & 6 & 6 & 6 \\
\hline Serovar 7 & $4-7$ & positive & 0 & 1 & 6 & 6 & 5 & 5 & 5 & 4 & 5 \\
\hline \multirow[t]{3}{*}{ WF83 } & & suspect & 0 & 4 & 0 & 0 & 0 & 0 & 0 & 0 & 0 \\
\hline & & negative & 6 & 1 & 0 & 0 & 0 & 0 & 0 & 0 & 0 \\
\hline & & no. pigs & 6 & 6 & 6 & 6 & 5 & 5 & 5 & $5^{\dagger}$ & 5 \\
\hline Serovar 12 & 12 & positive & 0 & 0 & 0 & 0 & 0 & 1 & 1 & 1 & 0 \\
\hline \multirow[t]{3}{*}{ 9799/84 } & & suspect & 0 & 0 & 0 & 0 & 0 & 0 & 0 & 0 & 0 \\
\hline & & negative & 6 & 6 & 6 & 6 & 6 & 5 & 5 & 5 & 5 \\
\hline & & no. pigs & 6 & 6 & 6 & 6 & 6 & 6 & 6 & 6 & $6^{\dagger}$ \\
\hline
\end{tabular}

${ }^{*}$ APP LPS ELISA interpretation based on optical density (OD): negative OD $<0.30$, suspect OD $0.30-0.39$, positive OD $\geq 0.40$

${ }^{\dagger}$ Missing data on one pig 
Table 3. Actinobacillus pleuropneumoniae ApxIV serum antibody ELISA (S/P) response over time

\begin{tabular}{|c|c|c|c|c|c|c|c|c|c|c|}
\hline & & \multicolumn{9}{|c|}{ Day post inoculation } \\
\hline Inoculum & Isotype & 0 & 7 & 14 & 21 & 28 & 35 & 42 & 49 & 56 \\
\hline Serovar 1 & $\operatorname{IgM}$ & 0.02 & 0.23 & $0.43^{*}$ & $0.29^{*}$ & $0.27^{*}$ & $0.35^{*}$ & $0.47^{*}$ & $0.41^{*}$ & $0.29^{*}$ \\
\hline \multirow[t]{2}{*}{ ATCC 27088} & $\operatorname{IgA}$ & 0.03 & 0.08 & 0.12 & 0.14 & 0.19 & 0.18 & 0.11 & 0.34 & $0.37^{*}$ \\
\hline & $\mathrm{IgG}$ & -0.03 & -0.00 & 0.09 & $0.12^{*}$ & $0.29^{*}$ & $0.49^{*}$ & $0.47^{*}$ & $0.58^{*}$ & $0.53^{*}$ \\
\hline Serovar $5 \mathrm{a}$ & IgM & 0.12 & 0.34 & 0.29 & 0.23 & 0.17 & 0.19 & 0.32 & 0.17 & 0.31 \\
\hline \multirow[t]{2}{*}{ ATCC 33377} & $\operatorname{IgA}$ & 0.05 & 0.11 & 0.16 & 0.17 & 0.19 & $0.35^{*}$ & $0.53^{*}$ & 0.31 & 0.29 \\
\hline & IgG & -0.02 & -0.00 & -0.01 & -0.00 & -0.00 & -0.00 & 0.01 & 0.00 & 0.02 \\
\hline \multirow{3}{*}{$\begin{array}{l}\text { Serovar } 7 \\
\text { WF83 }\end{array}$} & IgM & 0.19 & 0.20 & $1.32^{*}$ & $0.73^{*}$ & $0.44^{*}$ & $0.38^{*}$ & $0.35^{*}$ & $0.33^{*}$ & $0.25^{*}$ \\
\hline & IgA & -0.01 & 0.00 & 0.03 & 0.07 & 0.14 & 0.11 & 0.16 & 0.08 & 0.05 \\
\hline & $\mathrm{IgG}$ & -0.02 & -0.00 & 0.24 & $0.36^{*}$ & $0.38^{*}$ & $0.46^{*}$ & $0.44^{*}$ & $0.63^{*}$ & $0.73^{*}$ \\
\hline \multirow{3}{*}{$\begin{array}{l}\text { Serovar } 12 \\
9799 / 84\end{array}$} & IgM & 0.01 & 0.02 & 0.02 & 0.05 & 0.18 & 0.23 & 0.09 & 0.14 & 0.17 \\
\hline & IgA & 0.19 & 0.27 & 0.44 & 0.39 & 0.29 & 0.08 & 0.27 & 0.42 & 0.18 \\
\hline & IgG & -0.2 & -0.02 & -0.02 & -0.02 & 0.02 & 0.02 & -0.02 & -0.02 & -0.03 \\
\hline
\end{tabular}

${ }^{*}$ Within serovars, S/P responses were significantly higher than DPI 0 responses (ANOVA, $\mathrm{P}<0.05$ )

Table 4. Actinobacillus pleuropneumoniae ApxIV oral fluid antibody ELISA (S/P) response over time

\begin{tabular}{|c|c|c|c|c|c|c|c|c|c|c|}
\hline & & \multicolumn{9}{|c|}{ Day post inoculation } \\
\hline Inoculum & Isotype & 0 & 7 & 14 & 21 & 28 & 35 & 42 & 49 & 56 \\
\hline Serovar 1 & $\operatorname{IgM}$ & 0.05 & 0.07 & 0.06 & 0.15 & 0.05 & 0.04 & 0.04 & 0.02 & 0.02 \\
\hline \multirow[t]{2}{*}{ ATCC 27088} & $\operatorname{Ig} \mathrm{A}$ & 0.07 & 0.16 & 0.10 & 0.12 & 0.11 & 0.10 & 0.13 & 0.12 & 0.19 \\
\hline & $\mathrm{IgG}$ & 0.00 & 0.01 & -0.00 & $0.17^{*}$ & $0.23^{*}$ & $0.24^{*}$ & $0.42^{*}$ & $0.25^{*}$ & $0.21^{*}$ \\
\hline Serovar 5a & IgM & 0.07 & 0.03 & 0.06 & 0.06 & 0.13 & 0.05 & 0.05 & 0.03 & 0.02 \\
\hline \multirow[t]{2}{*}{ ATCC 33377} & $\operatorname{Ig} \mathrm{A}$ & 0.03 & 0.04 & 0.05 & 0.05 & 0.10 & 0.08 & 0.20 & 0.06 & 0.08 \\
\hline & IgG & -0.01 & -0.01 & -0.00 & 0.00 & 0.00 & -0.00 & 0.00 & -0.01 & -0.00 \\
\hline Serovar 7 & IgM & 0.09 & 0.13 & $0.39^{*}$ & 0.11 & 0.13 & 0.17 & 0.05 & 0.06 & 0.07 \\
\hline \multirow{2}{*}{ WF83 } & IgA & 0.00 & 0.23 & $0.25^{*}$ & 0.11 & 0.30 & $0.56^{*}$ & $0.46^{*}$ & $0.39^{*}$ & $0.47^{*}$ \\
\hline & IgG & -0.03 & 0.06 & $0.17^{*}$ & $0.23^{*}$ & $0.29^{*}$ & $0.60^{*}$ & $0.43^{*}$ & $0.41^{*}$ & $0.47^{*}$ \\
\hline \multirow{3}{*}{$\begin{array}{l}\text { Serovar } 12 \\
9799 / 84\end{array}$} & IgM & 0.04 & 0.11 & 0.06 & 0.06 & 0.05 & 0.07 & 0.05 & 0.02 & 0.02 \\
\hline & $\operatorname{Ig} \mathrm{A}$ & 0.04 & 0.10 & 0.08 & 0.08 & 0.09 & 0.12 & 0.08 & 0.05 & 0.07 \\
\hline & $\mathrm{IgG}$ & 0.01 & 0.01 & 0.01 & 0.01 & 0.00 & 0.01 & 0.00 & -0.00 & -0.00 \\
\hline
\end{tabular}

${ }^{*}$ Within serovars, S/P responses were significantly higher than DPI 0 responses (ANOVA, P $<0.05$ )

ApxIV ELISA oral fluid antibody responses. The ApxIV oral fluid antibody (IgG) ELISA S/P responses varied among serovars (Table 4). In serovar 1, the IgG $\mathrm{S} / \mathrm{P}$ responses were higher than DPI 0 on DPI 15 and later $(p=0.0096)$. In serovar $5, \operatorname{IgG~S} / \mathrm{P}$ responses higher than DPI 0 were detected at DPI $41(\mathrm{p}=0.0267)$. In serovar $7, \mathrm{~S} / \mathrm{P}$ responses greater than DPI 0 were detected at DPI 14 and later $(\mathrm{p}<0.0313)$. In serovar 12 , no difference in IgG response was found at any sampling point when compared to DPI 0 .

Comparisons between serovars showed that the magnitude of the $\operatorname{IgG~S} / \mathrm{P}$ antibody response differed between serovars 1 and 7 at DPI $22(p=0.0229)$, DPI 35 $(\mathrm{p}=0.0013)$ and later. The serovar $1 \mathrm{IgG} \mathrm{S} / \mathrm{P}$ response was higher than serovars 5 and 12 at DPI 16 and later $(p<0.0403)$. Likewise the serovar 7 response was higher than serovars 5 and 12 at DPI 15 and later $(\mathrm{p}<0.0361)$. No difference was detected in serovar 5 and 12 responses over time.

In serovar 1 , the IgM S/P responses were higher than DPI 0 at DPI $29(p=0.0157)$. In serovar 5, IgM S/P responses were higher that DPI 0 at DPI 3 through 11 $(\mathrm{p}=0.0355)$, and at DPI $45(\mathrm{p}=0.0163)$ through 56 $(\mathrm{p}=0.0161)$. In serovar 7, IgM responses were higher than DPI 0 at DPI $13(p=0.0261)$, DPI $14(p=0.0157)$, and DPI $15(\mathrm{p}<0.0001)$. In serovar 12 , no difference in IgM response was found at any sampling point when compared to DPI 0.

Comparisons between serovars showed that the serovar $7 \mathrm{IgM} \mathrm{S} / \mathrm{P}$ response was higher than serovars 1 , 5 , and 12 at DPIs 11 through $15(\mathrm{p}<0.0488)$ and then higher than serovar 1 at DPI $43(\mathrm{p}=0.0143)$ and DPI46 $(\mathrm{p}<0.0001)$. The serovar $1 \mathrm{IgM} \mathrm{S} / \mathrm{P}$ response was higher than serovar 12 at DPI $29(\mathrm{p}=0.0139)$. No difference was detected in serovar 5 and 12 responses over time when compared to each other.

In serovar 1 , the IgA $\mathrm{S} / \mathrm{P}$ responses were higher than DPI 0 at DPI $44(p=0.0482), 52(p=0.0371)$, and DPI $55(\mathrm{p}<0.0001)$. In serovar 7 , IgA responses were higher from DPI 0 at DPIs 10 and $11(\mathrm{p}=0.0307)$, then on DPIs $14-19(p<0.0218)$, DPI $30-56(p<0.0001)$. No detectable differences were found on serovars 5 and 12 over time when compared with DPI 0.

Comparisons between serovars detected no differences in the $\operatorname{IgA} \mathrm{S} / \mathrm{P}$ responses among serovars1, 5 , and 12 at any sampling point. The serovar $7 \mathrm{IgA} \mathrm{S} / \mathrm{P}$ responses was higher than serovars 1,5 , and 12 at DPI 23 and later $(\mathrm{p}<0.0001)$.

\section{Discussion}

The goal of this study was to describe ApxIVspecific antibody responses in serum and oral fluid from pigs inoculated with $A$. pleuropneumoniae under experimental conditions. As ApxIV is only produced by A. pleuropneumoniae, it is produced by all serovars and is highly immunogenic, i.e., produces detectable levels of serum antibodies in pigs $(8,12,21)$. ApxIV antibody offers the potential to monitor A. pleuropneumoniae infections in the field. Detection of ApxIV antibody has not been previously described in swine oral fluids, 
although oral fluid has been shown to be a suitable specimen for the detection of antibodies against a variety of pathogens, as reviewed elsewhere $(27,46)$.

The outcome of inoculating pigs with A. pleuropneumoniae under experimental conditions depends on the route and dose $(1,49)$. Several routes of A. pleuropneumoniae inoculation have been described, including intranasal (25, 36), endotracheal (1), endobrochial (26), direct application of the inoculum to the tonsils of the soft palate (7), and aerosol (19). Reproducing $A$. pleuropneumoniae infections under experimental conditions is complicated by the fact that lower challenge doses may not result in infection (54, 55 ). On the other hand, higher doses may cause severe clinical signs and/or acute death (47).

As distinct from the inoculation protocol used in this and other experimental studies, A. pleuropneumoniae infections in the field are believed to occur by direct contact between pigs or via aerosol exposure $(11,17)$. However, recent research has shown that the environment (drinking water, feed, soil) is also heavily seeded with $A$. pleuropneumoniae (32), suggesting that field infections may involve repeated low-dose exposures (55). Thus, differences among experimental results may reflect variability in the exposure route and dose under experimental conditions $(24,25,54,55)$ and differences between experimental results and field observations may result from the fact that experimental exposures do not reflect the mode of A. pleuropneumoniae transmission (dose, route, frequency) in the field (20).

In the present study, animals were inoculated both intranasally and by direct application of the inoculum to the tonsils of the soft palate. The production of clinical signs was marked and prolonged in the two groups (serovars 1 and 7) exposed to A. pleuropneumoniae at a concentration of $1 \times 10^{6} \mathrm{CFU} / \mathrm{ml}$ and mild or absent in groups exposed to $A$. pleuropneumoniae at a concentration of $1 \times 10^{5} \mathrm{CFU} / \mathrm{ml}$ (serovars 5 and 12).

The kinetics of the LPS-specific serum antibody response has been examined in previous studies. After aerosol exposure to serovar 1, LPS-specific IgM was reported at $14 \mathrm{DPI}, \mathrm{IgA}$ at 14 to $21 \mathrm{DPI}$, and IgG at 21 DPI (5). Following IN exposure to serovar 9, LPSspecific IgM was detected at 7 DPI and both IgA and IgG at 14 DPI (29). In the current study, the LPS serum antibody response for serovars 1 and 7 was in agreement with previous reports. Specifically, an LPS-specific IgG response was detected as early as 7 DPI, with all animals seropositive by DPI 14. Thereafter, all animals remained seropositive through DPI 56. Thus, the LPS serum antibody ELISA response was compatible with "productive" A. pleuropneumoniae infection in animals inoculated with serovars 1 and 7 . In contrast, a weak and transient LPS ELISA response was observed in animals inoculated with serovar 5 . In serovar 12 , a single animal was LPS ELISA positive on DPI 35, 42, and 49. These results are compatible with previous studies reporting variable success at infecting pigs with A. pleuro- pneumoniae under experimental conditions $(9,10)$, even at very high exposure doses $(40,53)$.

There are relatively few studies on ApxIV serum antibody ontogeny and these are based on the detection of ApxIV-specific IgG. Following aerosol exposure, ApxIVIgG was reported to be detected at 7 to 28 DPI in pigs inoculated with serovars $1,5 b, 6,7,10$, or 15 (12). In another study, seroconversion to ApxIV was reported at 3 to 6 weeks post inoculation in pigs exposed to serovars 1,7 , or 15 by either IN or endotracheal routes (13). In the current study, using the ApxIV ELISA S/P results from DPI 0 as the basis for comparison, a significant increase $(\mathrm{p}<0.05)$ in ApxIV antibody was detected in serum at $14 \mathrm{DPI}(\operatorname{IgM})$ and $21 \mathrm{DPI}(\operatorname{IgG})$ in animals inoculated with serovars 1 and 7 . This pattern was consistent with previous reports $(12,13)$, and also followed the pattern observed for the LPS ELISA response in these animals.

The results of this study suggested that the S/P cutoff of the commercial serum ApxIVIgG ELISA is too high. That is, using the manufacturer's recommended cutoff $(\mathrm{S} / \mathrm{P} \geq 0.50)$, "seroconversion" was delayed in some animals inoculated with serovars 1 or 7 up to DPI 49. However, a limitation of the current study was the small number of animals. As a consequence, it was not possible to conduct a valid statistical analysis of test performance, e.g., receiver operating characteristic analysis, to re-calculate the ELISA cutoff and associated test sensitivity and specificity.

In a previous report $A$. pleuropneumoniae $\operatorname{IgA}$ was detected in stimulated oral fluid samples collected 14 DPI after the IN inoculation of pigs with serovar 2 (33), but there are no previous reports of ApxIV antibody in swine oral fluid. Using the oral fluid ApxIV ELISA S/P results from DPI 0 as the basis for comparison, a significant increase $(p<0.05)$ in ApxIVIgG S/P ratios was detected at $\geq 21$ DPI in pigs inoculated with serovar 1 , with no detectable increase in IgM or IgA. In serovar 7, significantly higher $\mathrm{IgGS} / \mathrm{P}$ ratios were observed at $\geq 14$ DPI. In this group, a significant increase $(\mathrm{p}<0.05)$ in the ApxIV IgM response was observed at 14 DPI and in the IgA response at DPI 21 and then at $\geq 35$ DPI.

Respiratory diseases are among the most important issues in swine health worldwide. Among other determinants, the increasing intensification of pork production and the development of large, technified pig farms have created conditions suitable for the spread of agents associated with swine respiratory diseases (34). The development of tools for monitoring respiratory infections is a key component in the control of these diseases. Previous studies have demonstrated the efficiency of swine oral fluids for the detection of antibody against ASFV (38), influenza A virus (41), porcine circovirus type $2(45)$, porcine reproductive and respiratory syndrome virus (28), and others. Based on the data presented herein, it can be concluded that assays for detecting A. pleuropneumoniae ApxIV antibody in oral fluids could also be developed for monitoring A. pleuropneumoniae infections in commercial herds. 
Conflict of Interests Statement: The authors declare the following potential conflicts of interest with respect to the research authorship, and/or publication of this article: authors S. Lizano and C. Goodell are employed by IDEXX Laboratories, Inc. The remaining authors declare that there is no conflict of interests regarding the publication of this article.

Financial Disclosure Statement: This study was supported in part by Pork Check off funds distributed through the Iowa Pork Producers Association (Des Moines, Iowa, USA).

Animal Rights Statement: The study was approved by the Iowa State University Office for Responsible Research.

\section{References}

1. Baarsch M.J., Foss D.L., Murtaugh M.P.: Pathophysiologic correlates of acute porcine pleuropneumonia. Am J Vet Res 2000, 61, 684-690

2. Blackall P.J., Klaasen H.L.B.M., Van Den Bosch H., Kuhnert P., Frey J.: Proposal of a new serovar of Actinobacillus pleuropneumoniae: serovar 15. Vet Microbiol 2002, 84, 47-52.

3. Bochev I.:Porcine respiratory disease complex (PRDC): a review. I. Etiology, epidemiology, clinical forms and pathoanatomical features. Bulg J Vet Med 2007, 10, 131-146.

4. Bossé J.T., Janson H., Sheehan B.J., Beddek A.J., Rycroft A.N., Kroll J.S., Langford P.R.: Actinobacillus pleuropneunomiae: pathobiology and pathogenesis of infection. Microb Infect 2002, 4, 225-235.

5. Bossé J.T., Johnson R.P., Nemec M., Rosendal S.: Protective local and systemic antibody responses of swine exposed to an aerosol of Actinobacillus pleuropneumoniae serotype 1. Infect Immun 1992, 60, 479-484.

6. Bossé J.T., Li Y., Sárközi R., Gottschalk M., Angen Ø., Nedbalcova K., Rycroft A.N., Fodor L., Langford P.R.: A unique capsule locus in the newly designated Actinobacillus pleuropneumoniae serovar 16 and development of a diagnostic PCR. J Clin Microbiol 2017, doi:10.1128/JCM-02166.

7. Chiers K., Haesebrouck F., Van Overbeke I., Charlier G., Ducatelle R.: Early in vivo interactions of Actinobacillus pleuropneumoniae with tonsils of pigs. Vet Microbiol 1999, 68, 301-306.

8. Cho W.S., Chae C.:Expression of the ApxIV gene in pigs naturally infected with Actinobacillus pleuropneumoniae. J Comp Pathol 2001, 125, 34-40.

9. Costa G., Oliveira S., Torrison J., Dee S.: Evaluation of Actinobacillus pleuropneumoniae diagnostic tests using samples derived from experimentally infected pigs. Vet Microbiol 2011, 148, 246-251.

10. Costa G., Oliveira S., Torrison J.: Detection of Actinobacillus pleuropneumoniae in oral-fluid samples obtained from experimentally infected pigs. J Swine Health Prod 2012, 20, 78-81.

11. Desrosiers R., Moore C.: Indirect transmission of Actinobacillus pleuropneumoniae. J Swine Health Prod 1998, 6, 263-265.

12. Dreyfus A., Schaller A., Nivollet S., Segers R.P., Kobisch A.M., Mieli L., Sørensen V., Hüssy D., Miserez R., Zimmermann W., Inderbitzin F.: Use of recombinant ApxIV in serodiagnosis of Actinobacillus pleuropneumoniae infections, development and prevalidation of the ApxIV ELISA. Vet Microbiol 2004, 99, 227-238.

13. Eamens G.J., Gonsalves J.R., Whittington A.M., Turner B.: Evaluation of serovar-independent ELISA antigens of
Actinobacillus pleuropneumoniae in pigs, following experimental challenge with $A$. pleuropneumoniae, Mycoplasma hyopneumoniae and Pasteurella multocida. Aust Vet J 2012, 90, 225-234.

14. Frey, J.: Detection, identification and subtyping of Actinobacillus pleuropneumoniae. Methods Mol Biol 2003, 216, 87-95.

15. Giménez-Lirola L.G., Jiang Y.H., Sun D., Hoang H., Yoon K.J., Halbur P.G., Opriessnig T.: Simultaneous detection of antibodies against Apx toxins ApxI, ApxII, ApxIII, and ApxIV in pigs with known and unknown Actinobacillus pleuropneumoniae exposure using a multiplexing liquid array platform. Clin Vaccine Immunol 2014, 21, 85-95.

16. Giménez-Lirola L.G., Xiao C.T., Zavala M., Halbur P.G., Opriessnig T.: Improving ante-mortem diagnosis of Erysipelothrix rhusiopathiae infection by use of oral fluids for bacterial, nucleic acid, and antibody detection. J Microbiol Methods 2013, 92, 113-121.

17. Gottschalk M.: Actinobacillosis. In: Diseases of Swine, edited by J.J. Zimmerman, L. Kariker, A. Ramirez, K. Schwartz, G. Stevenson, Wiley-Blackwell, Hoboken, New Jersey, 2012, pp. 653-669.

18. Gottschalk M., De Lasalle F., Radacovici S., Dubreuil J.D.: Evaluation of long chain lipopolysaccharides (LC-LPS) of Actinobacillus pleuropneumoniae serotype 5 for the serodiagnosis of swine pleuropneumonia.Vet Microbiol 1994, 38, 315-327.

19. Grøndahl-Hansen J., Barfod K., Klausen J., Andresen L.O., Heegaard P.M., Sørensen V.: Development and evaluation of a mixed long-chain lipopolysaccharide based ELISA for serological surveillance of infection with Actinobacillus pleuropneumoniae serotypes 2, 6 and 12 in pig herds. Vet Microbiol 2003, 96, 41-51.

20. Hensel A., Stockhofe-Zurwieden N., Ganter M., Petzoldt K.: Aerosol exposure of pigs to viable or inactivated Actinobacillus pleuropneumoniae serotype 9 induce antibodies in bronchoalveolar lining fluids and serum, and protects against homologous challenge. Vet Microbiol 1995, 47, 27-41.

21. Huang H., Zhou R., Chen M.L., Liu J.J., Xu X.J., Chen H.C.: Cloning and expression of the ApxIVA gene of Actinobacillus pleuropneumoniae and development of an indirect ApxIVAELISA. Chin J Biotechnol 2005, 21, 131-136.

22. InzanaT.J.: Virulence properties of Actinobacillus pleuropneumoniae. Microb Pathog 1991, 11, 305-316.

23. Inzana T.J., Fenwick B.: Serologic detection of Actinobacillus pleuropneumoniae in swine by capsular polysaccharide-biotinstreptavidin enzyme-linked immunosorbent assay. J Clin Microbiol 2001, 39, 1279-1282.

24. Jacobsen M.J., Nielsen J.P., Nielsen R.: Comparison of virulence of differenct Actinobacillus pleuropneumoniae serotypes and biotypes using an aerosol infection model. Vet Microbiol 1996, 49, 159-168.

25. Jobert J.L., Savoye C., Cariolet R., Kobisch M., Madec F.: Experimental aerosol transmission of Actinobacillus pleuropneumoniae to pigs. Can J Vet Res 2000, 64, 21-26.

26. Kamp E.M., Stockhofe-Zurwieden N., Van Leengoed L.A., Smits M.A.: Endobronchial inoculation with Apx toxins of Actinobacillus pleuropneumoniae leads to pleuropneumonia in pigs. Infect Immun 1997, 65, 4350-4354.

27. Kittawornrat A., Prickett J., Chittick W., Wang C., Engle M., Johnson J., Patnayak D., Schwartz T., Whitney D., Olsen C., Schwartz K., Zimmerman J.: Porcine reproductive and respiratory syndrome virus (PRRSV) in serum and oral fluid samples from individual boars: will oral fluid replace serum for PRRSV surveillance? Virus Res 2010, 154, 170-176.

28. Kittawornrat A., Prickett J., Wang C., Olsen C., Irwin C., Panyasing Y., Ballagi A., Rice A., Main R., Johnson J., Rademacher C., Hoogland M., Rowland R., Zimmerman J.: Detection of porcine reproductive and respiratory syndrome virus (PRRSV) antibodies in oral fluid specimens using a commercial PRRSV serum antibody ELISA. J Vet Diagn Invest 2012, 24, 262-269.

29. Krejci J., Nechvatalova K., Kudlackova H., Faldyna M., Kucerova Z., Toman M.: Systemic and local antibody responses after 
experimental infection with Actinobacillus pleuropneumoniae in piglets with passive or active immunity. J Vet Med 2005, 52, 190-196.

30. Lacouture S., Mittal K.R., Jacques M., Gottschalk M.: Serotyping Actinobacillus pleuropneumoniae by use of monoclonal antibodies. J Vet Diagn Invest 1997, 9, 337-341.

31. Leary S., Underwood W., Anthony R., Cartner S., Corey D., Grandin T., Greenacre C.B., Gwaltney-Bran S., McCrackin M.A., Meyer R., Miller D.: Methods of Euthanasia. In: $A V M A$ Guidelines for the Euthanasia of Animals. American Veterinary Association, Schaumburg, Illinois (USA), 2013, pp. 18-41.

32. Loera-Muro V.M., Loera-Muro A., Morfin-Mata M., Jacques M., Avelar-González F.J., Ramírez-Castillo F., Ramírez-López E.M., Guerrero-Barrera A.L.: Porcine respiratory pathogens in swine farms environment in Mexico.Open J Anim Sci 2014, 4, 196-205.

33. Loftager M.K., Eriksen L., Nielsen R.: Antibodies against Actinobacillus pleuropneumoniae serotype 2 in mucosal secretions and sera of infected pigs as demonstrated by an enzyme-linked immunosorbent assay. Res Vet Sci 1993, 54, $57-62$.

34. Losinger W.C., Bush E.J., Smith M.A., Corso B.A.: Mortality attributed to respiratory problems among finisher pigs in the United States. Prev Vet Med 1998, 37, 21-31.

35. Markey B., Leonard F., Archambault M., Cullinane A., Maguire D.: General procedures in microbiology. In: Clinical Veterinary Microbiology. Mosby Elsevier, St. Louis, Missouri (USA), 2013, pp. 3-79.

36. Marois C., Gottschalk M., Morvan H., Fablet C., Madec F., Kobisch M.: Experimental infection of SPF pigs with Actinobacillus pleuropneumoniae serotype 9 alone or in association with Mycoplasma hyopneumoniae. Vet Microbiol 2009, 135, 283-291.

37. McFarland J.: The nephelometer: an instrument for estimating the number of bacteria in suspensions used for calculating the opsonic index and for vaccines. J Am Med Assoc 1907, 49, 1176-1178.

38. Mur L., Gallardo C., Soler A., Zimmermman J., Pelayo V., Nieto R., Sánchez-Vizcaíno J.M., Arias M.: Potential use of oral fluid samples for serological diagnosis of African swine fever.Vet Microbiol 2013, 165, 135-139.

39. Nielsen R., van den Bosch J.F., Plambeck T., Sørensen V., Nielsen J.P.: Evaluation of an indirect enzyme-linked immunosorbent assay (ELISA) for detection of antibodies to the Apx toxins of Actinobacillus pleuropneumoniae. Vet Microbiol 2000, 71, 81-87.

40. Opriessnig T., Hemann M., Johnson J.K., Heinen S., GiménezLirola L.G., O’Neill K.C., Hoang H., Yoon K.J., Gottschalk M., Halbur P.G.: Evaluation of diagnostic assays for the serological detection of Actinobacillus pleuropneumoniae on samples of known or unknown exposure. J Vet Diagn Invest 2013, 25, 61-71.

41. Panyasing Y., Goodell C.K., Giménez-Lirola L., Kittawornrat A., Wang C., Schwartz K.J., Zimmerman J.J.: Kinetics of influenza A virus nucleoprotein antibody (IgM, $\operatorname{IgA}$, and $\operatorname{IgG})$ in serum and oral fluid specimens from pigs infected under experimental conditions. Vaccine 2013, 31, 6210-6215.

42. Perry M.B., Altman E., Brisson J.R., Beynon L.M., Richards J.C.: Structural characteristics of the antigenic capsular polysaccharides and lipopolysaccharides involved in the serological classification of Actinobacillus (Haemophilus) pleuropneumoniae strains.Serodiagn Immunother Infect Dis 1990, 4, 299-308.

43. Pohl S., Bertschinger H.U., Frederiksen W., Mannheim W.: Transfer of Haemophilus pleuropneumoniae and the Pasteurellalike organism causing porcine pleuropneumonia to the genus Actinobacillus (Actinobacillus pleuropneumoniae comb. nov.) on the basis of phenotypic and deoxyribonucleic acid relatedness. Int J System Bacteriol 1983, 33, 510-514.

44. Pomorska-Mól M., Dors A., Kwit K., Kowalczyk A., Stasiak E., Pejsak Z.: Kinetics of single and dual infection of pigs with swine influenza virus and Actinobacillus pleuropneumoniae. Vet Microbiol 2017, 201, 113-120.

45. Prickett J.R., Johnson J., Murtaugh M.P., Puvanendiran S., Wang C., Zimmerman J.J., Opriessnig T.: Prolonged detection of PCV2 and anti-PCV2 antibody in oral fluids following experimental inoculation. Transbound Emerg Dis 2011, 58, 121-127.

46. Prickett J.R., Kim W., Simer R., Yoon K.J., Zimmerman J.: Oralfluid samples for surveillance of commercial growing pigs for porcine reproductive and respiratory syndrome virus and porcine circovirus type 2 infections. J Swine Health Prod 2008, 16, 86-91.

47. Rogers R.J., Eaves L.E., Blackall P.J., Truman K.F.: The comparative pathogenicity of four serovars of Actinobacillus (Haemophilus) pleuropneumoniae. Aust Vet J 1990, 67, 8-11.

48. Schaller A.,Kuhn R., Kuhnert P., Nicolet J., Anderson T.J., MacInnes J.I., Segers R.P., Frey J.: Characterization of ApxIV, a new RXT determinant of Actinobacillus pleuropneumoniae. Microbiology 1999, 145, 2105-2116.

49. Sebunya T.N., Saunders J.R., Osborne A.D.: Dose response relationship of Hemophilus pleuropneumoniae aerosol in pigs. Can J Vet Res 1983, 47, 54-56.

50. Shope R.E.:Porcine contagious pleuropneumonia. I. Experimental transmission, etiology and pathology. J Exp Med 1964, 119, 357-368.

51. Straw B.E., Shin S.J., Yeager A.E.: Effect of pneumonia on growth rate and feed efficiency of minimal disease pigs exposed to Actinobacillus pleuropneumoniae and Mycoplasma hyopneumoniae. Prev Vet Med 1990, 9, 287-294.

52. Veterinary Diagnostic Laboratory (VDL) Iowa State University. Procedure for standard plate count; standard operative procedure ( $2^{\text {nd }}$ version). Document No. 9.344, Ames, IA, USA, 2014

53. Tobias T.J., Bouma A., Daemen A.J., Wagenaar J.A., Stegeman A., Klinkenberg D.:Association between transmission rate and disease severity for Actinobacillus pleuropneumoniae infection in pigs. Vet Res 2013, 44, 2.

54. Van Leengoed L.A., Kamp E.M.: Endobronchial inoculation of various doses of Haemophilus (Actinobacillus) pleuropneumoniae in pigs. Am J Vet Res 1989, 50, 2054-2059.

55. Velthuis A.G.J., De Jong M.C.M., Stockhofe N., Vermeulen T.M.M., Kamp E.M.: Transmission of Actinobacillus pleuropneumoniae in pigs is characterized by variation in infectivity. Epidemiol Infect 2002, 129, 203-214. 